\title{
GRADENIGO'S SYNDROME AND PETROUS APICITIS: A RARE COMPLICATION OF COM: CASE REPORT
}

\author{
Lt. Col. Supreet Singh Nayyar1, Arvind Kumar Gupta ${ }^{2}$
}

${ }_{1}^{1}$ Graded Specialist, Department of Otorhinolaryngology, ENT Military Hospital, Ahmedabad. ${ }^{2}$ Resident, Department of Otorhinolaryngology, ENT Armed Forces Medical College.

\begin{abstract}
Gradenigo syndrome (Gradenigo-Lannois Syndrome) was described in 1907 by Giuseppe Gradenigo when he reported a triad of symptoms characterised by periorbital unilateral pain due to trigeminal nerve involvement at Meckel's cave, diplopia due to involvement of abducens nerve in Dorello's canal and persistent otorrhoea of involved ear. The classical triad has become very rare after the antibiotic era. Petrositis is an uncommon complication of otitis media. When it does occur; however, it can be devastating complication. The absence of natural drainage pathway with bottle neck to drainage posed by the bony labyrinth, which occupies the base of petrous pyramid and close proximity of CNS make petrous a dangerous site of infection. The diagnosis is made on clinical suspicion and can be usually confirmed by HRCT temporal bone. Management incudes parenteral antibiotics and surgical drainage with the objective to provide adequate drainage from the suppurative focus in the petrosa without damage to facial nerve or labyrinth. There are various approaches for drainage, i.e. infralabyrinthine, infracochlear, translabyrinthine, transcochlear, etc. We report here a case of Gradenigo syndrome with classical triad of deep periorbital pain, otorrhoea and diplopia due to lateral rectus palsy in a 55 years female with no known comorbidities presented to our OPD. She was managed actively with intravenous antibiotics and surgical drainage of petrous apex by infralabyrinthine approach.
\end{abstract}

\section{KEYWORDS}

Gradenigo's Syndrome, Petrous Apicitis.

HOW TO CITE THIS ARTICLE: Nayyar LCSS, Gupta AK. Gradenigo's syndrome and petrous apicitis: a rare complication of COM: case report. J. Evolution Med. Dent. Sci. 2016;5(74):5517-5519, DOI: 10.14260/jemds/2016/1246

\section{INTRODUCTION}

Gradenigo syndrome (Gradenigo-Lannois Syndrome) was described in 1907 by Giuseppe Gradenigo when he reported a triad of symptoms characterised by periorbital unilateral pain due to trigeminal nerve involvement at Meckel's cave, diplopia due to involvement of abducens nerve in Dorello's canal and persistent otorrhoea of involved ear. The classical triad has become very rare after the antibiotic era.

Petrositis is an uncommon complication of otitis media. When it does occur, however, it can be devastating complication. The absence of natural drainage pathway with bottle neck to drainage posed by the bony labyrinth, which occupies the base of petrous pyramid and close proximity of CNS make petrous a dangerous site of infection.

The diagnosis is made on clinical suspicion and can usually confirmed by HRCT temporal bone.

Management incudes parenteral antibiotics and surgical drainage with the objective to provide adequate drainage from the suppurative focus in the petrosa without damage to facial nerve or labyrinth. There are various approaches for drainage i.e. infralabyrinthine, infracochlear, translabyrinthine, transcochlear, etc.

We report here a case of Gradenigo syndrome with classical triad of deep periorbital pain, otorrhoea and diplopia due to lateral rectus palsy in a 55 years female with no known comorbidities presented to our OPD. She was managed

Financial or Other, Competing Interest: None.

Submission 07-08-2016, Peer Review 01-09-2016,

Acceptance 07-09-2016, Published 15-09-2016.

Corresponding Author:

Dr. Lt. Col. Supreet Singh Nayyar,

Graded Specialist,

Department of Otorhinolaryngolgy,

Military Hospital,

Ahmedabad.

E-mail: ssnayyar@gmail.com

DOI: $10.14260 /$ jemds $/ 2016 / 1246$ actively with intravenous antibiotics and surgical drainage of petrous apex by infralabyrinthine approach.

\section{CASE REPORT}

A 55 yr. female with no known comorbidities presented to our OPD with complaints of ear discharge left side of four months' duration, deep seated hemifacial pain and diplopia of four days' duration. There was no history of vertigo, tinnitus or aural fullness. No history of fever, neck stiffness, seizure suggestive of intracranial complication. There was no other ENT complaint. On examination-patient was conscious, alert, oriented with GCS 15/15. Vitals were stable. Ear findings-left small central perforation on anteroinferior quadrant of pars tensa with pulsatile discharge. Discharge was mucopurulent, not foul smelling, not blood stained. Rest of pars tensa was thickened scarred with landmarks not clearly defined. Tuning for test showed mild conductive hearing loss on left side. Otoneurological test showed abducens nerve palsy left side. There was no spontaneous or gaze nystagmus, no sign of facial nerve involvement. Fistula test was negative. No sign of cerebellar involvement.

HRCT temporal bone showed soft tissue attenuation mass in the left epitympanum and mesotympanum, superomedially over the round window niche and oval window. The mass is also noted in the Prussak's space with mild blunting of the scutum. Posterosuperiorly, there is extension into the mastoid air cells. The left tympanic membrane is retracted. Facial nerve and lateral semicircular canal is intact. No evidence of bony erosion. Evidence of poor pneumatisation of mastoid air cells on left side. Middle ear ossicles is normal.

MRI brain $(\mathrm{P}+\mathrm{C})$ abnormal signal intensity is seen in left mastoid air cells, left petrous apex and left sided Meckel's cave. It appears hyperintense on T2W, FLAIR and hypointense on T1W. On post contrast, it shows predominantly peripheral enhancement. It is abutting the petrous portion of the left ICA. Cavernous sinus appear normal, suggestive of left mastoiditis and petrositis with soft tissue extending to left Meckel's cave. 
No focal lesion or abnormal enhancement seen in brain parenchyma.

The patient was immediately started on IV antibiotic covering gram positive and gram negative organism.

She underwent cortical mastoidectomy and drainage of petrous apex via infralabyrinthine approach under general anaesthesia. Intraoperative finding-antrum was filled with hypertrophic mucosa and granulation tissue. Facial nerve canal was identified and preserved, semicircular canal preserved. Petrous apex reached and decompressed. No pus was found. Ossicles were found to be intact and mobile. All ossicles were preserved. Postop was uneventful, IV antibiotic continued for fourteen days. Patient is doing well.

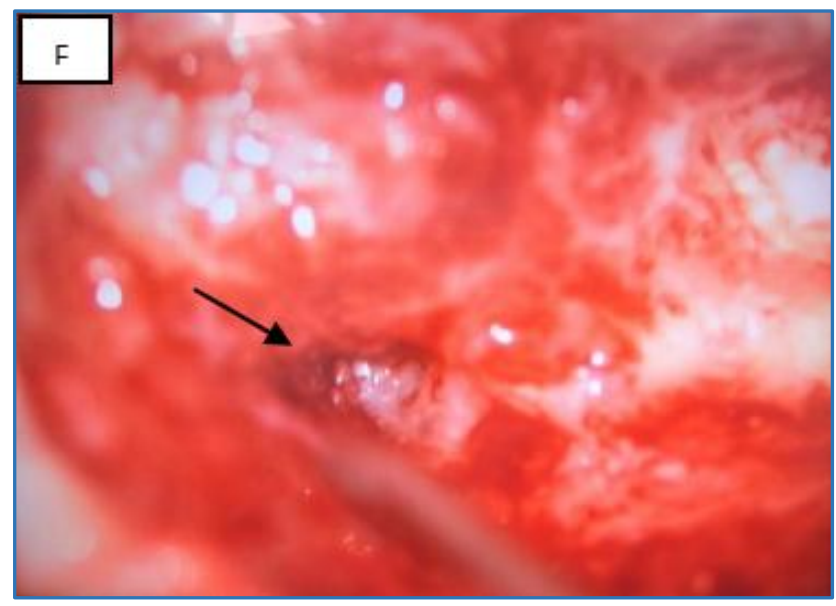

Fig. 1: Intraoperative Left Ear showing Petrous Apex (Arrow)

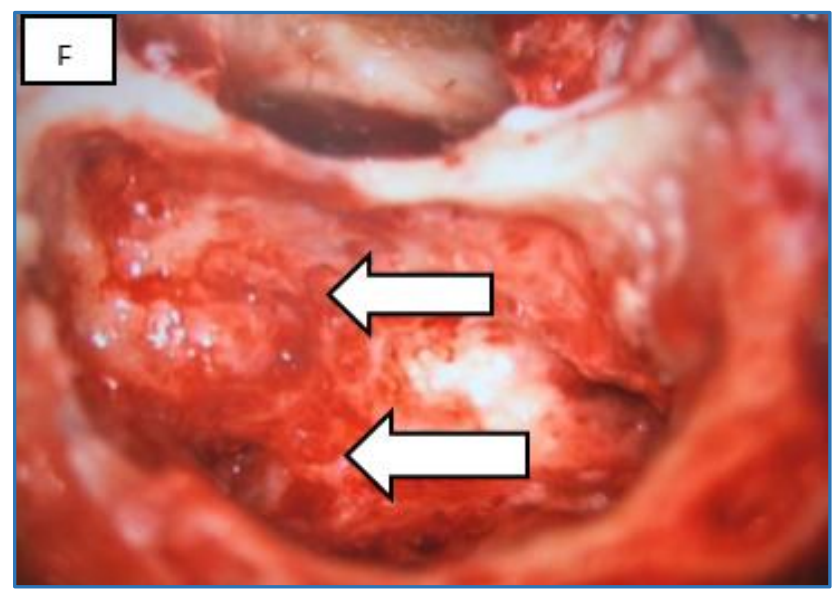

Fig. 2: ILT - Infralabyrinthine Tunnel for Petrous Apex

\section{DISCUSSION}

The trigeminal ganglion and the sixth cranial nerve are separated from the bony petrous apex only by the dura mater, hence their vulnerability to inflammatory processes occurring within this region. ${ }^{(1)}$

Like mastoid, petrous bone is of three types: pneumatised, diploic and sclerotic. Pneumatisation of petrous apex occurs only in $30 \%$ of cases with cells extending from the middle ear or mastoid to the petrous apex; $4-7 \%$ Pneumatisation is asymmetrical. With less than one-third of petrous bone are pneumatised into the apex. In our case, pneumatisation of mastoid on affected side was poor.
Pathways for spread of infection are described by cell tracts from mastoid to petrous apex. These are namely posterosuperior, posteromedial, sub-arcuate, peritubal and perilabyrinthine. Posterosuperior tract which starts in the mastoid and runs behind or above the bony labyrinth to the petrous apex, subarcuate pass through the arch of superior semicircular canal to reach the apex. Infective process runs along these cell tracts and reaches the petrous apex.

In case of posterior petrositis the pain is occipital, parietal, or temporal, and the discharge is from the mastoid and in case of anterior petrositis the pain is frontal or behind the eye and discharge is from the tympanum. Our patient presented with symptoms of pain along the distribution of trigeminal nerve, otorrhoea and diplopia.

Inflammation spread can lead to meningitis, empyema, brain abscess, venous thrombosis, cranial nerve paralysis and internal carotid artery stenosis. $(2,3)$ Time lapse between otological symptoms and cranial nerve dysfunction varies from 1 to 12 weeks. $(2,4)$ In our case, it was 12 to 16 weeks.

Less common symptoms of petrositis include transient facial paresis, mild recurrent vertigo and fever usually low grade and intermittent. CT scan is the first choice of imaging, since it is widely available and detects changes in bone structures including destruction of trabecular bone and erosion of the petrous apex.(5,6) MRI is more sensitive in detecting dural thickening and enhancement as well as intracranial complications. An MRI angiography may be performed to rule out signs of sinus thrombosis.(5)

Treatment shifts from conservative to surgical management depending on patient's presenting stage, presence or absence of complications and response to treatment. Conservative management includes broadspectrum antibiotics from three to six weeks. Our patients showed immediate pain relief on day 1 of antibiotic. Gradenigo's syndrome is most commonly caused by aerobic microorganisms, but it may also be found in interaction with anaerobic microorganisms. Surgical drainage should be done in impending complication to ensure adequate disease clearance.

\section{Possible Sequel of Gradenigo's Syndrome}

- Meningitis.

- Intracranial abscess.

- Spread to skull base and involvement of IX, X XI cranial nerve (Vernet's syndrome).

\section{Aetiology of Gradenigo's Syndrome}

- Apical Petrosis secondary to otitis media.

- Extra dural inflammation at petrous apex involving trigeminal ganglion and abducens nerve.

\section{Diagnostic Criteria of Gradenigo's Syndrome}

- Suppurative otitis media.

- Pain in the distribution of trigeminal nerve.

- Abducens nerve palsy.

\section{REFERENCES}

1. Kantas I, Papadopoulou A, Balatsouras DG, et al. Therapeutic approach to Gradenigo's syndrome: a case report. J Med Case Rep 2010;4:151.

2. Guedes V, Gallegos P, Ferrero A, et al. Gradenigo's syndrome: a case report. Arch Argent Pediatr 2010; 108(3):e74-5. 
3. Kong SK, Lee IW, Goh EK, et al. Acute otitis media induced petrous apicitis presenting as the Gradenigo syndrome: successfully treated by ventilation tube insertion. Am J Otolaryngol 2011;32(5):445-7.

4. Marianowski R, Rocton S, Ait-Amer JL, et al. Conservative management of gradenigo syndrome in a child. Int J Pediatr Otorhinolaryngol 2001;57(1):79-83.
5. Luntz M, Brodsky A, Nusem S, et al. Acute mastoiditis-the antibiotic era: a multicenter study. Int J Pediatr Otorhinolaryngol 2001;57(1):1-9.

6. Gibier L, Darrouzet V, Franco-Vidal V. Gradenigo syndrome without acute otitis media. Pediatr Neurol 2009;41(3):215-9. 